\title{
Seroprevalence of Swine Salmonellosis in Korean Swine Herds
}

\author{
Yeong-Hun Kim, III-Kyong Kwon', and Jeong-Hee Han* \\ School of Veterinary Medicine and Institute of Veterinary Science, Kangwon National University, \\ Chuncheon 200-701, Korea \\ ${ }^{1}$ College of Animal Resources Science, Kangwon National University, Chuncheon 200-701, Korea
}

\begin{abstract}
Salmonellosis is one of the most important wasting diseases that leads to economic damage in the swine industry. Many risk factors have been reported to increase the spread of Salmonella infection; therefore, it is important to understand how to treat the risk factors of Salmonella to effectively prevent salmonellosis in commercial pig farms. To accomplish this, we conducted a study to determine if the seasons and porcine production stages affected the serological response to Salmonella in Korea. A total of 1,592 serum samples submitted to the School of Veterinary Medicine of Kangwon National University between Jan. 2001 and Dec. 2004 from commercial farms were tested by ELISA. The overall apparent seroprevalence of salmonellosis was 38.1\% (95\% CI, 38.0-38.2), while the prevalence of Salmonella according to seasons and production stages ranged from $17.9 \%$ to $62.8 \%$ for the former $(24.6 \%$ in spring, $17.9 \%$ in summer, $38.5 \%$ in autumn, and $62.8 \%$ in winter) and from $16.1 \%$ to $68.3 \%$ for the latter (17.9\% in suckling pigs, $16.1 \%$ in weaning pigs, $37.50 \%$ in growers, $41.9 \%$ in finishers, $48.0 \%$ in gilts, and $68.3 \%$ in sows). In this study of seroprevalence by production stage, most pigs were naturally infected by Salmonella during the weaning stage. Also, seroprevalences were found to have a seasonal pattern in which most pigs were infected in autumn to winter.
\end{abstract}

Key words: swine salmonellosis, seroprevalence, ELISA, Korean swine herd

\section{Introduction}

Salmonella Enterica is an important and well-recognized food-borne pathogen in humans, but it also induces severe economic losses in terms of swine mortality (Kishima et al., 2008; Thorns et al., 2000). Although the more than 2,400 Salmonella serotypes categorized according to Kouffman-white scheme have various host ranges and are spread extensively, the primary clinical disease in pigs is caused by $S$. Choleraesuis or $S$. Typhimurium belonging to serogroups B and C, respectively (Griffith et al., 2006; Yoshida et al., 2007). Pigs can be infected with a variety of $S$. enterica serovars and infected pigs can be healthy carriers, spreading infection from both the intestinal tract and the mesenteric lymph nodes for extended periods of time (Griffith et al., 2006).

The status and types of feed, housing contamination, antibacterial usage, presence of rodents and cats, herd

\footnotetext{
*Corresponding author : Jeong-Hee Han, School of Veterinary Medicine and Institute of Veterinary Science, Kangwon National University, Chuncheon 200-701, Korea. Tel: 82-33-2508691, Fax: 82-33-256-3722, E-mail: hanjh@kangwon.ac.kr
}

health status, etc are considered as risk factors affecting the high prevalence of salmonellosis (Funk and Gebreyes, 2004). It is difficult to determine common risk factors due to differences in production, industry structure, and regulatory organization (Funk and Gebreyes, 2004). However, in a number of advanced countries, especially European countries, Salmonella reduction programs have been applied and proven to be effective (Christensen and Rudemo, 1998). Others, such as Italy, are investigating the epidemiology of salmonellosis for the future implementation of a reduction program (Merialdi et al., 2008). Nevertheless, some countries are continuously conducting risk factor studies as monitoring the prevalence of salmonellosis is important to prevent from salmonellosis and to make decisions on control strategies (Sanchez et al., 2007).

However, in Korea, there have been few reports of salmonellosis risk factors to achieve reasonable accuracy over long periods of time. The development of efficient strategies to control swine salmonellosis would help to decrease the presence of Salmonella. Therefore, we have conducted intensive longitudinal studies via ELISA, with the aim of determining and describing the correlation 
between the degree of infection with porcine production stages and seasons.

\section{Materials and Methods}

\section{Study population}

A total of 1,592 serum samples submitted to the School of Veterinary Medicine of Kangwon National University between Jan. 2001 and Dec. 2004 from commercial farms were evaluated for the prevalence of Salmonella. The samples were divided into groups of different production stages including suckler ( $<22 \mathrm{~d}$ old), weaner (22-70 days old), grower (71-119 d old), finisher (>120 days old), gilt and sow, and of different seasons, including spring (March-May), summer (June-August), autumn (September-November) and winter (December-Feburary). All serums submitted were heated for $30 \mathrm{~min}$ at $56^{\circ} \mathrm{C}$ for complement inactivation. And then the individual samples were stored below $-20^{\circ} \mathrm{C}$ until use.

\section{ELISA}

The samples were slowly defrosted in a refrigerator between 4 and $7^{\circ} \mathrm{C}$ in preparation for the Salmonella ELISA (HerdCHek* Swine Salmonella Test Kit, IDEXX, USA). The coating antigens in this ELISA include LPS (lipopolysaccharide) of serogroup B, C1 and D (O-antigens $1,4,5,6,7$, and 12). The ELISA protocol was carried out according to the manufacturer's instructions (wavelength: $650 \mathrm{~nm}$ ). OD values of samples, positive and negative control were determined by measuring at $650 \mathrm{~nm}$. An S/P > 0.5 or greater according to below calculation was considered to be a positive serum antibody response. The ELISA test has a specificity of $99.4 \%$.

$$
\begin{aligned}
\mathrm{S} / \mathrm{P} \text { ratio }= & (\text { Sample OD }- \text { Negative OD }) / \\
& (\text { Positive OD }- \text { Negative OD })
\end{aligned}
$$

\section{Statistical analysis}

Data gathered from the survey were entered into a Microsoft Excel (Redmond, WA, USA) spreadsheet. The analysis was performed using the glimmix macro in Statistical Analysis System (SAS Institute, Inc., Cary, $\mathrm{NC}$, USA) and the provincial differences for seroprevalence of salmonellosis in Korean pigs were compared using the chi-square test according to season and production stages $(p<0.05)$.

\section{Results}

\section{Prevalence by season}

The relationship between Salmonella seroprevalence and season in Korea from 2001 to 2004 was shown in Table 1. A total of 607 pigs (38.1\%; 95\% CI, 38.0-38.2) out of 1,592 tested were positive for Salmonella by ELISA. The seasonal sero-prevalence of Salmonella spp. ranged from $17.86 \%$ to $68.29 \%$; $24.6 \%$ (95\% CI, 24.025.2 ) in spring, $17.9 \%$ (95\% CI, 17.2-18.6) in summer, $38.5 \%$ (95\% CI, 38.1-38.9) in autumn and 62.8\% (95\% CI, 62.6-63.0) in winter. Seroprevalence of Salmonella in serum decreased gradually between spring and summer, and increased considerably from $17.9 \%$ which is the lowest rate in summer until it reached a peak of $62.8 \%$ in winter $(X=209.2317, p<0.0001)$.

\section{Prevalence by production stages}

The results of Table 2 revealed Salmonella seroprevalence in the following age categories: 0 to $21 \mathrm{~d}$ (suckler), 21 to $70 \mathrm{~d}$ (weaner), 70 to $119 \mathrm{~d}$ (grower), $120 \mathrm{~d}$ and above (finisher), gilt and sow. The seropositivity rates in suckler, weaner, grower, finisher, gilt, and sow are $23.7 \%$

Table 1. Seroprevalence of Salmonella by season in Korean pigs

\begin{tabular}{lccc}
\hline \hline \multirow{2}{*}{ Season } & \multicolumn{3}{c}{ Individual survey } \\
\cline { 2 - 4 } & $\begin{array}{c}\text { Selected } \\
\text { samples (A) }\end{array}$ & $\begin{array}{c}\text { Positive } \\
\text { samples (B) }\end{array}$ & $\begin{array}{c}\text { B/A (\%) } \\
\left(95 \% \mathrm{CI}^{1)}\right)\end{array}$ \\
\hline Spring & 370 & 91 & $24.6(24.0-25.2)$ \\
Summer & 336 & 60 & $17.9(17.2-18.6)$ \\
Fall & 413 & 159 & $38.5(38.1-38.9)$ \\
Winter & 473 & 297 & $62.8(62.6-63.0)$ \\
\hline \multicolumn{1}{c}{ Total } & 1592 & 607 & $38.1(38.0-38.2)$ \\
\hline 1) $95 \%$ confidence interval. & & \\
$\chi^{2}=209.2317, p<0.0001$. & &
\end{tabular}

Table 2. Seroprevalence of Salmonella according to production stages in Korean pigs

\begin{tabular}{cccc}
\hline \hline \multirow{2}{*}{$\begin{array}{c}\text { Production } \\
\text { stages }\end{array}$} & $\begin{array}{c}\text { Selected } \\
\text { samples (A) }\end{array}$ & $\begin{array}{c}\text { Positive } \\
\text { samples (B) }\end{array}$ & $\begin{array}{c}\text { B/A (\%) } \\
\left(95 \% \mathrm{CI}^{1)}\right)\end{array}$ \\
\cline { 2 - 4 } Suckler & 270 & 64 & $23.7 \%(23.5-23.9)$ \\
Weaner & 311 & 50 & $16.1 \%(16.0-16.2)$ \\
Grower & 347 & 130 & $37.5 \%(37.3-37.7)$ \\
Finisher & 217 & 91 & $41.9 \%(41.6-42.2)$ \\
Gilt & 177 & 85 & $48.0 \%(47.6-48.4)$ \\
Sow & 270 & 187 & $69.3 \%(69.1-69.5)$ \\
\hline Total & 1,592 & 607 & $38.1 \%(38.0-38.2)$ \\
\hline 95\% confidence interval. & & \\
$\chi^{2}=207.5812, p<0.0001$. & &
\end{tabular}


(95\% CI, 23.5-23.9), 16.1\% (95\% CI, 16.0-16.2), 37.5\% (95\% CI, 37.3-37.7), $41.9 \%$ (95\% CI, 41.6-42.2), $48.0 \%$ (95\% CI, 47.6-48.4), and 69.3\% (95\% CI, 69.1-69.5), respectively. Seroprevalence dropped between suckler (23.7\%; 95\% CI, 23.5-23.9) and weaner $(16.1 \%$; 95\% CI, 16.0-16.2) stages, but then increased dramatically to the highest peak of $69.3 \%$ at the sow stage, 4 times higher than the weaner stage $(X=207.5812, p<0.0001)$.

\section{Discussion}

In Korea, Hazard Analysis Critical Control Point (HACCP) has established performance standards to reduce the prevalence of Salmonella in swine through onfarm interventions (Kim et al., 2008). However, there is no useable data on the prevalence of Salmonella and official control program in pigs in Korea.

The estimate of Salmonella prevalence for a specific period could be a good indicator of how well salmonellosis control measures are working on farms (Funk et al., 2000). Here, we performed ELISA to measure the prevalence of Salmonella spp. because the prevalence of Salmonella has never been correlated with sero-positivity in Korea. The advantages of ELISAs for detecting pigs infected with salmonellosis over other methods include greatly improved sensitivity, the low cost per sample, and the ability to test large numbers of samples (Leotides et al., 2003). However, the ELISA kit used in this study could only detect pigs infected with serogroups B, C1 and D of Salmonella spp. or pigs that had already produced antibodies from a prior Salmonella infection before sampling (van der Heijden, 2001).

The overall seroprevalence in our study was estimated at $38.13 \%$, even though ELISA could only detect the antibody for serogroups B, C1, and D of Salmonella. The incidences of Salmonella spp. detected from swine fecal samples in the USA and Japan were only $4.9 \%$ and 3.1\%, respectively (Bahnson et al., 2006; Kishima et al., 2008). In one Korean study, Salmonella incidence was reported at 9.5\% from fecal samples (Kim et al., 2001). Therefore, sampling design might be an important characteristic related to Salmonella prevalence documentation (Sanchez et al., 2007). In a previous Korean study, Salmonella was isolated from $139(21 \%)$ of 662 fecal samples as the result of bacterial culture on pigs with diarrhea or a history of diarrhea, which were lower results than the present study (Futagawa-Saito et al., 2008). This may be explained by a report comparing ELISA with bacterial culture, which indicated that a farm with a $50 \%$ preva- lence determined by the blood ELISA showed only as $39 \%$ prevalence based on fecal culture sample methods, and an even lower value of $16 \%$ prevalence based on cecum analysis (Sanchez et al., 2007). Therefore, it is probable that the difference between detecting methods might affect the prevalence results.

Suh and Jung (2005) reported the highest prevalence of Salmonella was in suckling/nursery pigs. However, David et al. (1998) reported that prevalence reached the highest level in breeding pigs. Our study revealed that antibodies of Salmonella were extensively distributed throughout all stages of swine growth, with seroprevalence reaching a peak in the sow stage and being lowest in weaner pigs.

The early period after birth is important for the health and development of swine. During this period, the susceptibility to infectious diseases is not only affected by a variety of factors, including maturity of the immune system and susceptibility to tolerogenic signals, but maternal antibodies, especially IgA, also plays a critical role to protect animals from the diarrheal diseases (Offit and Clark, 1985). In our results, the swine maternal antibody was responsible for the weaner pigs having the lowest level of Salmonella seropositivity. In addition, the dramatic increase of seroprevalence from weaner to grower may suggest that the highest number of infections break out between day 22 and day 70 of life. Improvement and application of health control during this period might be an important key point in controlling the spread of Salmonella. However, the continued increase of seroprevalence throughout all growth stages of swine showed that Salmonella infection was not limited only to the younger swine.

Jette et al. (1998) demonstrated that Danish swine had higher seroprevalence in winter than summer, which was similar to our results. Our results clearly indicated a seasonal pattern in salmonellosis outbreaks. In winter, it is not only difficult to control the ventilation and the temperature on farms, but the unpredictability of weather conditions makes proper setting of ventilation systems difficult, to create conditions that can cause stress and encouraging bacterial infection. The ventilation of swine barns is a compromise between maintaining adequate air exchange while conserving heat (Funk and Gebreyes, 2004). Swine farms equipped with the wrong ventilation system have reported a severe economic loss by dint of wasting diseases and faulty ventilation.

ELISAs have been proven to be a useful tool for the detection of farms contaminated with Salmonella, but it cannot give information on the infectious status of individual swine at a specific point in time (Leotides et al., 
2003). The results indicated by ELISA are different when polymerase chain reaction or bacterial cultures are used. Therefore, we will conduct further studies in the future regarding the difference of prevalence data collecting using these different detection tools.

In conclusion, the results of the prevalence pattern in this study indicate that the things associated with season and production stages could act as the risk factors provoking the increase of Salmonella seroprevalence.

\section{Acknowledgement}

This work was supported by a grant (20080401034019) from BioGreen 21 Program, Rural Development Administration, Republic of Korea.

\section{References}

1. Bahnson, P. B., Fedorka-Cray, P. J., Ladely, S. R., and Mateus-Pinilla, N. E. (2006) Herd-level risk factors for Salmonella enterica subsp. Enterica in U.S. market pigs. Prev. Vet. Med. 76, 249-262.

2. Christensen, J. and Rudemo, M. (1998) Multiple changepoint analysis applied to the monitoring of Salmonella prevalence in Danish pigs and pork. Prev. Vet. Med. 36, 131-143.

3. David, P. R., Bovie, F. G. M., Funk, J. A., Morrow, W. E. M., Jones, F. R., and Deen, J. E. (1998) Isolation of Salmonella serotypes from feces of pigs raises in multi-site production system in NC, USA. J. Am. Med. Assoc. 212, 1925-1929.

4. Funk, J. and Gebreyes, W. A. (2004) Risk factors associated with Salmonella prevalence on swine farms. J. Swine Health Prod. 12, 246-251.

5. Funk, J. A., Davies, P. R., and Nichols, M. A. (2000) The effect of fecal sample weight on detection of Salmonella Enterica in swine feces, J. Vet. Diagn. Invest. 12, 142-148.

6. Futagawa-Saito, K., Hiratsuka, S., Kamibeppu, M., Hirosawa, T., Oyabu, K., and Fukuyasu, T. (2008) Salmonella in healthy pigs: Prevalence, serotype diversity and antimicrobial resistance observed during 1998-1999 and 2004-2005 in Japan. Epidemiol. Infect. 136, 1118-1123.

7. Griffith, R. W., Schwartz, K. J., and Meyerholz, D. K. (2006) Salmonella. In Diseases of Swine ed. Straw, B. E., Zimmer- man, J. J., D'Allair, S., and Taylor, D. J. pp. 739-754. Ames: Blackwell Publishing Professional.

8. Kim, S. H., Chun, S. G., Park, M. S., Park, J. H., and Lee, B. K. (2008) Finding the sources of Korean Salmonella enterica Serovar Enteritidis PT4 isolates by pulsed-field gel electrophoresis. J. Microbiol. 46, 209-213.

9. Kim, S. Y., Lee, H. M., Kim, S., Hong, H. P., and Kwon, H. I. (2001) Epidemiological aspects of Salmonella spp. infections of domestic animals in Gyeongbuk province. Korean $J$. Vet. Serv. 24, 43-50.

10. Kishima, M., Uchida, I., Namimatsu, T., Osumi, T., Takahashi, S., Tanaka, K., Matsuura, K., and Yamamoto, K. (2008) Nationwide surveillance of Salmonella in the faeces of pigs in Japan. Zoonoses Public Health, 55, 139-44.

11. Leotides, L. S., Grafanakis, E., and Genigeorgis, C. (2003) Factors associated with the serological prevalence of Salmonella Enterica in Greek finishing swine herd. Epidemiol. Infect. 131, 599-606.

12. Merialdi, G., Barigazzi, G., Bonilauri, P., Tittarelli, C., Bonci, M., D'incan, M., and Dottori, M. (2008) Longitudinal study of Salmonella infection in Italian farrow-to-finish swine herds. Zoonoses Public Health, 55, 232-236.

13. Offit, P. A. and Clark, H. F. (1985) Protection against rotavirus induced gastroenteritis in a murine model by passively acquired gastrointestinal but no circulating antibodies. $J$. Virol. 54, 58-64.

14. Sanchez, J., Dohoo, I. R., Christensen, J., and Rajic, A. (2007) Factors influencing the prevalence of Salmonella spp. in swine farms: A meta-analysis approach. Prev. Vet. Med. 81, 148-177.

15. Suh, D. K. and Jung, S. C. (2005) Epidemiological characteristics of Salmonella spp. isolated from different stages of commercial swine farms. Korean J. Vet. Serv. 45, 179-183.

16. Thorns, C. J. (2000) Bacterial Foodborne Zoonoses, Rev. Sci. Tech. 19, 226-239.

17. van der Heijden, H. M. (2001) First international ring trial of ELISAs for Salmonella-antibody detection in swine. Berl. Munch. Tierarztl. Wochenschr. 114, 389-392.

18. Yoshida, C., Franklin, K., Konczy, P., McQuiston, J. R., Fields, P. I., Nash, J. H., Taboada, E. N., and Rahn, K. (2007) Methodologies towards the development of an oligonucleotide microarray for determination of Salmonella serotypes. J. Microbiol. Methods 70, 261-271.

(Received 2009.7.27/Revised 2010.2.16/Accepted 2010.2.17) 